\section{PRÁCTICAS DE CIUDADANÍA EUROPEA. EL USO ESTRATÉGICO DE LAS IDENTIDADES EN LA PARTICIPACIÓN POLIITICA DE LOS INMIGRANTES COMUNITARIOS*}

\author{
Michael Janoschka \\ Instituto de Economía, Geografía y Demografía - CSIC
}

\section{EUROPEAN CITIZENSHIP PRACTICE. THE STRATEGIC USE OF IDENTITIES AND POLITICAL PARTICIPATION OF EU MIGRANTS}

\begin{abstract}
Local politics can be evaluated as an outstanding field of negotiation and social exchange that emblematize the deep transformations of the Spanish society due to the massive migration processes of the last decade. This article debates the magnitude of political change that was introduced by granting immigrants (mainly EU citizen) with active and passive voting rights, analyzing empirically observed forms of political participation at the Mediterranean coastal areas. This analysis is conceptually framed through the debate of two theoretical notions, namely the role European citizenship and European Identity play for the transformation of local politics. Both ideas are conceptualized and empirically debated as practices of EU rights and capacities formed by collective action that refers to the "Idea" of Europe.
\end{abstract}

KEY WORDS: European citizenship; Political participation; Politics of Identity; Migration; Spain.

\section{A MODO DE INTRODUCCIÓN \\ El primer alcalde británico en España - ¿símbolo de una sociedad integradora?}

"Un inglés que no sabe español, alcalde accidental de San Fulgencio" ", titulaba el rotativo La Vanguardia el 31 de octubre de 2008, cuando, tras la detención de la alcaldesa, del teniente alcalde y de varios concejales por supuestos casos de corrupción urbanística, le tocaba asumir temporalmente el cargo de alcalde a un edil de origen británico que forma parte de la coalición gobernante de ese pueblo en la costa alicantina. La noticia, ampliamente difundida tanto en los medios españoles de cobertura nacional ${ }^{2}$ como en la prensa británica ${ }^{3}$, expone al público una transformación en la política local española que ha ido cobrando fuerza desde que a los extranjeros comunitarios se les
RESUMEN: La política local es un campo de negociación e intercambio social en el cual se perciben de forma destacada las profundas transformaciones que la sociedad española vivió a partir de los procesos masivos de inmigración de la última década. Este texto debatirá, a través de un análisis empírico de las formas de participación política en la costa mediterránea, qué alcance y magnitud de cambio introduce el derecho de voto activo y pasivo de extranjeros comunitarios. Para ese análisis, se efectúa un enlace conceptual entre la participación política a nivel local y algunas nociones teóricas como la ciudadanía europea. Los dos conceptos claves del debate, es decir, la ciudadanía europea y las referencias a identidades europeas, se piensan como una práctica diaria que se expresa activamente en cuestiones de indole político a nivel local.

PALABRAS CLAVE: Ciudadanía europea; Participación política; Políticas de identidad; Inmigración; España.

concediera el derecho de voto activo y pasivo en las elecciones municipales.

El año 1999, cuando por primera vez la vía de sufragio se abrió de forma generalizada a los extranjeros procedentes de países de la Unión Europea que viven en España ${ }^{4}$, marca el inicio de un cambio profundo en la geografía política de algunos municipios, localizados primordialmente en la costa mediterránea. A medida que España evolucionaba a lo largo de la última década hasta llegar a ser el país con la mayor dinámica inmigratoria de la Unión Europea ${ }^{5}$, extensas áreas de las provincias de Alicante y Málaga, y de las Islas Baleares y Canarias se han poblado, entre otros, de un contingente específico de extranjeros. En su gran mayoría provienen de los países de la Unión Europea (especialmente la "antigua" UE-15, más Suiza y Noruega), con Gran Bretaña y Alemania a la cabeza, y una parte importante de ellos 
son jubilados. A diferencia de las migraciones económicas, este flujo se caracteriza por el hecho de que sus protagonistas son personas con horizonte de vida amplio, que proceden de estratos sociales de alto poder adquisitivo y que migran por razones de ocio y con objeto de mejorar su calidad de vida (Rodríguez, et al., 2005). Recientemente, los efectos de esta migración específica, que en el debate anglo-sajón se denomina con los términos de "amenity migration" (Mclntyre 2009), "lifestyle migration" (Benson, 0'Reilly 2009) o "affluent migration" (Schriewer, Encinas 2009), ha sido objeto de un debate científico creciente (Echezarreta 2005; King, et al. 2000; Oliver 2007; Warnes 2004).

El pueblo inicialmente mencionado de 11.594 habitantes en 2008, San Fulgencio, es un reflejo de una nueva realidad que está emergiendo sin tener una repercusión mediática equiparable al flujo de migraciones económicas: según los datos del Padrón Municipal del año 2008, solamente el $23,1 \%$ de los habitantes de este municipio son de nacionalidad española. Por otro lado, los habitantes británicos aportan más de la mitad y junto con los alemanes constituyen casi dos tercios de la población municipal (INE 2008). Es posible, entonces, esperar que la participación política de extranjeros sea solamente un paso lógico en el proceso de apropiación cultural o integración social en el lugar de residencia, especialmente viviendo en un pueblo con semejante estructura demográfica. De acuerdo con las estadísticas, San Fulgencio forma parte de un grupo de municipios que presentan características poblacionales similares. En distintas localidades como Calpe, Rojales, Els Poblets (Alicante) y Mojácar (Almería), los extranjeros comunitarios exceden al número de españoles. Además, es un patrón bastante habitual en los municipios alicantinos y andaluces contar con una población de extranjeros comunitarios que supera el tercio del total y que esa población sea mayor de edad, con porcentajes superiores al 30\% del total de la población (INE 2008). Como consecuencia, y trasladado al campo político local, los extranjeros teóricamente podrian haber votado su propio concejal en más de 40 municipios andaluces, como afirman Durán y Martín (2008), refiriéndose a las elecciones del año 2003 cuando las cifras de la inmigración europea eran todavía marcadamente inferiores a las actuales. No sorprende que los comicios municipales de 2007 finalmente hayan ocasionado un ascenso significativo del número de concejales extranjeros, que solamente en la provincia de Alicante se elevan a tres docenas, repartidas primordialmente entre los municipios con mayor dinámica de la inmigración "acomodada" que se produce en el Levante español.

Esta primera indagación levanta un sinfín de cuestiones de indole sociológica acerca de las causas, los procesos y las consecuencias de la participación política de los extranjeros, sumamente interesantes desde un punto de vista tanto empírico como teórico. En este artículo se discutirá en qué medida la intervención activa en la política formal y la participación política a través del sufragio se pueden interpretar como una superación de las múltiples barreras sociales que viven y sufren extranjeros e inmigrantes de igual manera. A diferencia de algunos autores que discuten las vías "informales" de participación política a través de asociaciones y movimientos de indole transnacional (p.ej., Routledge 2008), se estudiará primordialmente en este trabajo la participación en la política formal. Ésta se puede caracterizar como uno de los campos significativos de ciudadanía, cuyo acceso está organizado a la vez de manera protegida y restrictiva (Ong 2006; Hermes y Dahlgren 2006; Shachar y Hirschl 2007). Algunos de los casos concretos que el autor ha investigado en los últimos años ${ }^{6}$ demostrarán los cambios que ha experimentado la política local a partir de la participación activa de los extranjeros comunitarios. Eso ayudará a entender en qué medida se puede explicar la participación política de extranjeros comunitarios como un signo de éxito de una sociedad integradora, tal como lo entiende el actual Plan Estratégico de Ciudadanía e Integración del Gobierno de España, es decir, como un "proceso bidireccional que busca la cohesión social" (MTAS 2007: 7). En este sentido, y frente a la incorporación de un contingente mayor de inmigrantes que podrá acudir a las urnas en los próximos comicios locales del año 2011 debido a la incorporación de los países del Este de Europa a la UE y los convenios bilaterales con países latinoamericanos como Argentina y Colombia, se pueden derivar conclusiones que tendrán cierta validez para la situación en muchos municipios en todo el país en un futuro muy próximo.

La interpretación de la participación política se efectuará en referencia a discusiones teóricas sobre la integración, uniendo dos aspectos fundamentales: la conceptualización de ciudadanía y de las identidades. Se defiende que, dada la actualidad y la intensidad del proceso de inserción política de los extranjeros comunitarios, la experiencia 
del Levante español genera resultados que no solamente resultan novedosos desde un punto empírico. Asimismo, se prevé un avance teórico-conceptual en los estudios relacionados con la ciudadanía que, como se verá en adelante, se interpreta como una práctica de políticas de identidad. En este sentido, cabe destacar que uno de los puntos de referencia son las variadas conceptualizaciones de Europa, tanto en cuestiones de identidad como en relación con la ciudadanía. Ese cuadro conceptual genera una perspectiva que atraviesa ciertos límites disciplinarios y a la vez incita a un relevo de la percepción que tiene el concepto de identidad europea, discutido en ocasiones como un ideario elitista y alejado de lo cotidiano de la población.

\section{Ciudadanía Europea y Políticas de identidad}

\section{Un croquis teórico-conceptual para el análisis de la participación política en un mundo transnacional}

Algunos de los debates recientes en teoría política y filosofía política destacan que la conceptualización de la ciudadanía está viviendo una transformación profunda, dado el nuevo y cambiado rol que tiene el estado-nación a partir de los procesos de globalización y la profundización de la Unión Europea (Bauböck 2005; Benhabib 2005; Habermas 2009). En este sentido, parece que algunos de los conceptos tradicionales, por ejemplo el de ciudadanía, definida primordialmente a través de los derechos civiles, políticos y sociales (Marshall 1950), precisan de una reconsideración. Esto también atañe a los estudios de Hannah Arendt (1987) que describe las tradicionales dicotomías binarias entre la ciudadanía y la falta de nacionalidad, centrándose en los deberes y los derechos de la ciudadanía. Por el contrario, una parte creciente del debate intelectual actual enfatiza la noción de ciudadanía, sobre todo a través de sus expresiones en la vida diaria: por ejemplo, las movilizaciones y las prácticas concretas que conlleva (Isin y Wood 1999; Ong 2006). Ello no significa ninguna negación de los derechos de la ciudadanía, constituyentes e indiscutidos. Más bien, se trata de una ampliación de las perspectivas teóricas, dando así una respuesta a las múltiples transformaciones que han vivido las sociedades post-fordistas, con nuevas relaciones de poder económico y social concentrado en "ciudades globales" (Sassen 1999) y las respuestas del estado-nación en un mundo trans- nacional con múltiples y variados procesos de migración transnacional (Vandenberg 2000; Davidson 2000; Barnett 2003; Low 2004). Especialmente éste es el caso de la Unión Europea, en donde, y como consecuencia de la implementación del Tratado de Maastricht, se han producido una serie de transformaciones de índole económica, cultural, jurídica y política. Según algunos autores, el aumento de las libertades laborales, de movimiento y de residencia condujeron a la formación de un era post-nacional que incluye la construcción de una práctica de la sociedad civil europea y la elaboración de nuevas formas de gobernanza (Finke y Knodt 2005). Asimismo, se establecieron formas de ciudadanía novedosas que cuestionan su concepción tradicional basada de forma exclusiva en la nacionalidad (Wiener 1998). Los derechos europeos, como la posibilidad de votar y de ser candidato en elecciones municipales, se rigen primordialmente por dos factores: la nacionalidad de uno de los estados miembro de la UE y la pertenencia a un territorio local concreto (Day y Shaw 2002). Así, se promociona una forma de concebir la ciudadanía como una práctica social que incluye la participación política en ámbitos espaciales y sociales muy variados (Rose 2001). En definitiva, autores como Mitchell (2003: $397 \mathrm{f}$ ) constatan un proceso de una creciente de-consolidación nacional y defienden la desterritorialización de la participación democrática. Se argumenta, en consecuencia, que la movilidad creciente de los ciudadanos europeos y las posibilidades formales de participación política conducen de forma automática al desarrollo de una sociedad civil europea (Urry 2000, Rumford 2002).

Dentro de ese panorama, algunos ciudadanos, como los migrantes jubilados inicialmente mencionados que, según Mitchell (2007) y Nijman (2007) pueden ser denominados como cosmopolitas, parecen especialmente privilegiados por la posesión de una gran variedad de opciones de estilos de vida. Son un ejemplo vivo del desarrollo del mundo transnacional y se pueden caracterizar como los prototipos de "ciudadanos transnacionales" que establecen nuevas formas de poder social a distintos niveles (Faist 1998; Favell 2003). En última instancia, cabe resaltar que, según esa línea discursiva, las políticas urbanas y la participación política que posteriormente se analizarán, se convierten por excelencia en cuestiones de índole transnacional. Como resultado de los debates mencionados, se deriva que en un mundo globalizado una parte creciente de las relaciones sociales atraviesan las fronteras de los 
estados-nación (Jackson et al. 2004: 8). Las ideas centrales de las "geografías del transnacionalismo" distinguen tres perspectivas diferentes, Ilamadas transnacionalismo "desde arriba", "desde abajo" y "de por medio". Mientras que en el primer caso los estudios se asocian al impacto de las instituciones transnacionales para las sociedades en cuestión, el transnacionalismo "desde abajo" se entiende normalmente como la incorporación de diferentes formas de resistencia local, por ejemplo, a través de activismo local y organizaciones vecinales. Según Smith (2001), transnacionalismo "de por medio" se define como un producto de prácticas políticas y sociales que están en un permanente proceso de reconstrucción, incluyendo así las transformaciones de todo tipo de prácticas (políticas) diarias e individualizadas. Esta idea puede evaluarse como un intento de conceptualizar las transformaciones de prácticas individuales y su entorno social en un mundo global. Lepofsky y Fraser (2003) argumentan que una ciudadanía post-nacional, que responda a las transformaciones del mundo transnacional, debería incluir no solamente un mero repertorio de derechos y deberes. La ciudadanía participativa post-nacional, como se podría denominar el concepto, implica sobre todo la articulación de identidades y transforma ciudadanía desde un derecho constitucional a un acto performativo que se desarrolla a través de la participación en la sociedad. Dado que la participación primordialmente se observa en asuntos locales, García (2006) introduce el término de "ciudadanía urbana", mientras Isin y Wood (1999) se refieren al concepto de "ciudadanía local". En las sociedades europeas, con la posibilidad de referirse a los derechos transnacionales concedidos en el marco de la Unión Europea y de hacer uso del voto a escala municipal, parece evidente que se ha establecido una discusión extendida acerca de las consecuencias prácticas de la "nueva" ciudadanía europea, que se entiende como ciudadanía participativa relativamente avanzada para sus propios miembros (Penninx et al. 2004; Favell 2008; Painter 2008).

Como acentúan Isin y Wood (1999), la ciudadanía se nutre de una multitud de posibilidades de acción. Si se acepta su propuesta de que la ciudadanía es tanto un conjunto de derechos y deberes como una serie de prácticas culturales, económicas y simbólicas, es necesario asumir las consecuencias teóricas, políticas y sociales de ese punto de vista. Entonces, la ciudadanía se tendrá que concebir como un concepto que cambia en el transcurso del tiempo y que se utiliza estratégicamente para mantener, reconocer o expandir las exigencias de distintos grupos sociales. Concebido de tal manera, la ciudadanía es un objeto de permanente pugna y negociación e incluye la expresión de identidades colectivas. Concebir la ciudadanía como una práctica y una capacidad de actuar en un ambiente determinado, tal como lo proponen Rose (2001) o Wiener (1998), ayuda a acoplarla con ideas teóricas que otorgan importancia a la expresión de identidades (políticas). En referencia a los debates efectuados en la rama de los estudios culturales (Cultural Studies) británicos, Stuart Hall (1997) expresa que las políticas de identidad son un aspecto sumamente importante como expresión de la ciudadanía a nivel local.

Teniendo en cuenta que, por razones de disponibilidad temporal y por un mayor interés en cuestiones del entorno inmediato, los ciudadanos jubilados suelen involucrarse con mayor énfasis en prácticas de política local (Jennings y Markus 1988), cabe cuestionar de qué manera es posible observar esta práctica de ciudadanía entre los residentes extranjeros en la costa mediterránea española. La participación política implica participar en las decisiones acerca de la producción del espacio y se refiere a cuestiones de poder. Según Harvey (2006), es importante observar en qué condiciones especificas el contexto de gobernanza local permite o no a los extranjeros una mayor implicación política. Antes de dar respuesta a esa cuestión y para fomentar la comprensión del enlace conceptual entre ciudadanía e identidad, se continúa con el debate acerca de la relevancia que tiene la expresión de identidades en la política local.

\section{Politicas de IDENTIDAD Y PARTICIPACIÓN POLÍtICA DE EXTRANJEROS}

\section{Conceptualizar el uso estratégico de identidades en la política local}

En un mundo marcado por los procesos migratorios, la expresión de identidades es un aspecto de mayor interés, dado que refleja los procesos de construcción social tanto de grupos sociales como de individuos. Según ideas promovidas primordialmente desde la perspectiva de los estudios culturales, se destaca que las identidades per- 
sonales, colectivas y políticas son un constructo social y un posicionamiento relacional que de manera permanente sufre procesos de desestabilización (Hall 1996). Las identidades se inventan a través de las prácticas, las relaciones, las no-relaciones, las ausencias, los intersticios y otras localizaciones continuas en el mundo social, de forma más o menos consciente. El conocer, entender y construir identidades auto-relacionadas ("yo" y "nosotros") implica por lo menos un conocimiento tácito del "Otro" ("tú" y "ellos") que no forma parte de uno mismo y del grupo de referencia propio (Massey 2004; Penrose, Mole 2008). En consecuencia, la identidad siempre se establece en relación a un sistema de diferencias y a través de ellas (Hall 1996: 4). En otras palabras: "identity requires difference in order to be, and it converts difference into otherness in order to secure its own self-certainty" (Connolly 2002: XIV).

La aceptación de nociones de identidades relacionales, flexibles y desestabilizadas conllevan una cadena de comprensiones que son interesantes desde una perspectiva teórica y también política. Siguiendo a Massey (2004), la apreciación del des-centrado y de las multiplicidades internas de identidades implica una perspectiva radical que reta a pensar muchos conceptos sociales y espaciales como ciudad, región, nación o Europa, todos evaluados como formaciones discursivas con relaciones de poder implícitas e inscritas. Si entonces los grupos se conceptualizan como "comunidades imaginadas" (Anderson 2006 [1983]), ellas no deberian diferenciarse por su autenticidad supuesta, sino primordialmente por la forma hegemónica por la cual se imaginan y construyen (Wodak et al. 1999). Siguiendo argumentos de teóricos feministas y poscoloniales, el constructo social en el que se basan tanto las identidades de grupos como las de los individuos, normalmente tiende a desaparecer o ser invisible por los procesos de naturalización (Said 2003; Butler 1990). Otros autores como Mouffe (2002), coinciden que este proceso es un acto necesario y a la vez performativo dentro de las relaciones hegemónicas para poder establecer la posibilidad de representación.

Muchos de los debates relacionados con políticas de identidad se originaron alrededor de los procesos de construcción de identidades nacionales y su contextualización en el marco de identidades culturales, ambas comprendidas como construcciones históricamente contingentes. Las identidades nacionales siguen siendo un esquema importante de expresión de la adhesión colectiva a los grupos sociales construidos, pero el foco de atención se ha desplazado hacia representaciones alternativas para estudiar cuestiones de identidad. Teóricos poscoloniales como Bhabha (2008) respaldan la tesis de que los vínculos culturales se producen de manera performativa. En consecuencia, enfocan su análisis de las identidades en las formas en que los códigos culturales naturalizados se exponen, con el fin de desestabilizarlos. Sin embargo y a pesar de una variedad de estudios acerca del mundo social "híbrido", la cuestión de cómo se usan estratégicamente los conceptos culturales esenciales, por ejemplo, en la resistencia política o conflictos políticos, no ha sido abordada con la profundidad necesaria más allá de lo que se entiende por la necesidad de establecer fijaciones "frágiles" y temporales del significado que constituyen identidades (Glasze 2007). En este sentido, Hall (1997) ofrece dos posibles reacciones de políticas de identidad para responder a las pérdidas de importancia que sufre el estado-nación en el mundo globalizado. Por un lado, se trata de un nacionalismo defensivo y agresivo en búsqueda de la posibilidad de establecer nuevas relaciones de poder y dominación mediante el uso de identidades culturales ancladas en un pasado ficticio. La otra forma de hacer frente al desafío identitario en el mundo global puede ser a través de un proyecto integrador que desarrolla activamente nuevas formas de identificación cultural. Son precisamente estas nuevas maneras de identificación en el mundo posmoderno a las que se refiere la idea de las identidades transnacionales. Isin y Wood (1999) recurren a la simultaneidad y ambigüedad que implican lo performativo de las identidades, especialmente en referencia a la necesidad constante de afirmación y transcendencia. Según ellos, la aplicación de las políticas de identidad busca a la vez el reconocimiento colectivo y la dominación. Ciertamente, esto pasa en áreas tan variadas como las identidades de género y sexuales, las expresiones de estilo de vida y de gustos o asimismo la cultura de consumo y el hedonismo (Featherstone 1991). La migración de extranjeros jubilados y económicamente acomodados a España es también una expresión de un estilo de vida que se basa, entre otros hechos, en el consumo de comodidades como un entorno espacial atractivo y condiciones climáticas evaluadas de manera positiva y que comparte algunas ideas referidas a procesos de un envejecimiento "exitoso".

Ahora, para discutir la participación política de los mencionados residentes extranjeros en España, se requiere considerar dos aspectos relacionados con el uso de mar- 
cas de identidad. El primer aspecto hace referencia a que las identidades colectivas no pueden ser pensadas sin las relaciones de poder existentes, que siempre son una expresión de o una protesta en contra de ellas. Segundo, las identidades han de ser evaluadas como una representación motivada por el interés político que se basa comúnmente en un fundamento emocional compartido para activar los procesos de integración del grupo mismo. En este aspecto destaca explícitamente el significado de las políticas de identidad que promueven innovaciones sociales y políticas, desafiando los procesos tradicionales de identificación. Esto pasa al margen del hecho de que todos los procesos de acción política necesitan implícitamente fijaciones temporales de las identidades fluidas y flexibles. En actividades políticas en un entorno globalizado, con una variedad de apropiaciones y de significantes de carácter transnacional, las cuestiones de la identidad cultural juegan un papel decisivo. Si se entienden las políticas de identidad de esa forma, como representaciones de significados sociales y espaciales, se abre un abanico de coaliciones políticas y una base compartida para la acción concreta en conflictos políticos. Como lo destaca Nicholls (2009), los actores que participan en la política y en sus conflictos habituales proyectan identidades esenciales en función de silenciar la multitud de diferencias internas que puede provocar su participación -tanto a nivel individual como para el colectivo que representan-. Castiglione (2009) se refiere a eso mediante el uso del término de "identidad política". La identidad política incluye dos nociones distintas: Por un lado, incluye la conceptualización de la manera en que la acción política y las instituciones contribuyen al proceso de identificación y diferenciación. Esa noción abre la posibilidad de extender el sentimiento de pertenencia simultánea a diferentes grupos y formaciones sociales. Por otro lado, la identidad política también abarca el sentido de lealtad hacia la comunidad política para facilitar al grupo la identificación $y$, a la vez, la defensa contra amenazas externas. Este proceso puede, como se discutirá en el debate empírico de la participación de los extranjeros en las costas del Levante español, dar a luz a grupos políticos que se refieren a identidades políticas interpretadas culturalmente. Por otro lado, permite también la creación de identidades políticas incluyentes que, en este caso especifico, se refieren primordialmente a construcciones de Europa y de identidad europea. Dentro de las múltiples condiciones de globalización cultural, se trata aquí de una situación prototípica: los residentes extranjeros, en gran parte jubilados, al formar parte de una generación activamente implicada en "hacer Europa" en su sentido más amplio y a través de sus profesiones anteriores y su comportamiento residencial actual, encabezan en algunos pueblos el giro de una política que se dirige explícitamente a valores y creencias europeas.

\section{IDENTIDADES EUROPEAS - UN RECURSO ESTRATÉGICO EN LA ELABORACIÓN DE POLITICAS LOCALES}

\section{Conceptualizar el uso estratégico de identidades en la política local}

A diferencia de las investigaciones relacionadas con la cultura juvenil, la elaboración de nuevos estilos musicales, los aspectos subculturales o los movimientos sociales y políticos subversivos cuyo enfoque central se basa en las "nuevas" formas de apropiación cultural desde la base social (della Porta, Tarrow 2004; Martí, Bonet 2008; Nicholls 2009), la discusión de identidades europeas se suele referir a una aproximación que se considera más bien elitista. Ello se deriva primordialmente de su enfoque en aspectos como las instituciones europeas, los burócratas de la administración de la Unión o los profesionales transnacionales (Checkel, Katzenstein 2009; Hermann, Brewer 2004; Laffan 2004; Wodak 2004). Los diferentes estudios explican que las élites transnacionales presentan una integración y adaptación selectiva respecto a las estructuras existentes de poder y jerarquía. Esa adaptación ocurre rápidamente en el ámbito profesional, mientras que las prácticas diarias son un campo de un acomodo más bien lento y selectivo (Beaverstock 2005; Favell 2008). La participación política de los extranjeros comunitarios en el litoral español evidencia esa relación. Por un lado, a nivel político, se efectúa un uso estratégico y con determinación de algunos aspectos específicos dentro del ámbito colectivo, público y político de la identidad europea. Por otro lado, en lo que concierne al estilo de vida individual, pocas expresiones de identidad europea se pueden observar. En concreto, tanto las actividades diarias como la gran mayoría de las amistades se establecen con compatriotas y personas que dominan su lengua nativa. Esta discrepancia concede interés teórico a la conceptualización del uso estratégico de políticas de identidad europea en las luchas políticas locales, por ejemplo, en contra de los modus operandi en 
los debates y las tomas de decisión sobre el desarrollo local. Como el interés de participar en la política local suele partir de preocupaciones personales, se aprecia una fuerte personalización en las políticas identitarias, enfatizando entre otros aspectos una supuesta brecha entre lo que se evalúa como una diferencia culturalmente interpretada entre las políticas locales en España y el país de origen, diferencia que se postula en una relación de equivalencia con el constructo social de "Europa":

\begin{abstract}
"As someone coming from Britain and someone who used to work in local government in Britain, you bring with you a kind of European concept of democracy and fairness and consultation and honesty and trust with politicians and all of those. Even in Britain, politicians aren't always a hundred per cent trustworthy. But when you deal with them here long enough, what you realize is, that you have to clear your mind of any ideas that you brought with you from England or Germany or Holland, wherever you might come from, and start thinking Spanish because they just don't accept or understand any of those principles ${ }^{8}$." (Entrevista a M.H., activista político en la provincia de Alicante)
\end{abstract}

La cita se refiere explícitamente a discrepancias que se interpretan en términos culturales y que a pesar de ser caracterizado como "británico" o para generalizarlo más, de "cosmopolita" o de "Europa occidental", se expresan como supuestamente "europeo". Es una marca de identidad europea que se basa en las normas de una administración pública "moderna" y las formas de negociación política "moderna". Este tipo de exigencias de índole identitario y marcadas como "europeas" se ponen en escena mediante actividades colectivas e individuales con el fin de obtener transformaciones en las políticas urbanas. Como el ejemplo demuestra sin lugar a duda, las referencias a identidades europeas siguen las mismas construcciones que caracterizan todo tipo de políticas de identidad, es decir, el principio de establecer de forma discursiva categorias de identidad y diferencia, de inclusión y exclusión que establecen el lugar del "propio" y del "Otro" constituyente. Según Bauböck et al. (2003), los conceptos flexibles y dinámicos de identidad europea reflejan las influencias de la globalización cultural y la forma de construir identidad en sociedades postmodernas, basadas en la comunicación mediática. En consecuencia y también como resultado de la ausencia de una esfera pública europea y de una narrativa hegemónica, las representaciones de Europa se idean como un concepto de mayor controversia que en el caso de las identidades nacionales, a su vez sobrecargadas tanto simbólica como histórica y políticamente. En este sentido, Amin (2004) se centra en su análisis en las dinámicas implícitas en la idea de Europa, tanto por los migrantes transnacionales como por la elite económica europea. Estos actores globalizan y organizan un espacio social híbrido que está íntimamente conectado con el estilo de vida crecientemente transnacional por parte de las clases medias -un aspecto que representa una objeción contra las perspectivas descritas que evalúan Europa y el proceso de europeización como una visión meramente elitista y burocrática (Checkel, Katzenstein 2009)-. La tesis de Amin se basa en la convicción que Europa se está desarrollando hacia un "place of plural and strange belongings, drawing in varied geographies of cultural formation ${ }^{\prime \prime}$ (Amin 2004, 2). Los argumentos presentados conducen a la conclusión de que todo intento de representar la identidad europea como algo claro y bien definido está destinado a naufragar. Explicitan el conflicto entre el significado y la condición de vaguedad que pueden ser evaluados como elementos constitutivos de todas las identidades de una Europa cultural y geográficamente ambigua.

Ahora bien, las perspectivas constructivistas ofrecen un campo conceptual interesante para debatir las políticas de identidad. Desde un punto de vista teórico, es sumamente estimulante que las identidades europeas puedan nutrirse de un sinnúmero de contenidos. Pero, dentro del marco de las prácticas diarias y los usos estratégicos de las políticas de identidad (europea), solamente una cantidad reducida de contenido se vuelve relevante. Si analizamos estos usos y esas apropiaciones, la diferencia entre "nosotros" y "ellos" resulta un punto de partida razonable. Según Eder (2006), las proposiciones acerca de la consistencia de la identidad europea dan por sentado esa distinción, organizándola dentro de una secuencia trascendental que genera una fidelidad narrativa para los sujetos participantes. En este sentido, Grundy y Jamieson (2007) evalúan la identidad europea como una categoría vacía que, por su contenido ambiguo, no es capaz de integrar ni a individuos ni a grupos. Esta percepción contradice a teóricos sociales como Habermas (1998), que se refiere al "patriotismo constitucional" y a la pasión que ciudadanos activos y activistas de movimientos políticos desarrollan para proteger los derechos humanos recurriendo a principios "europeos". En este sentido, el uso activo de las instituciones europeas 
puede ser una expresión de esa ciudadanía democrática, y algunos movimientos transnacionales de protesta se adaptan estratégicamente a las interpretaciones de principios universales definidos en textos constitucionales y por las instituciones de la Unión Europea (Kantner 2006). En consecuencia, las políticas de protesta social se refieren de forma creciente a esas nuevas relaciones de poder brindadas por los principios democráticos de Europa y de la UE para solucionar conflictos locales y regionales. Es en esta relación en la que se basa la expresión, algo imprecisa, del activista entrevistado, cuando menciona "el concepto europeo de democracia, de juego limpio, de consulta mutua y honestidad". Como veremos más adelante, el término de "democracia" es el que prima sobre los principios de integración identitaria, cuando se trata de identidades europeas. Según Mikkeli (1998), el campo semántico que confina a las identidades europeas se basa primordialmente en aspectos como la civilización, el cristianismo, la libertad, la piel blanca, el occidente $y$, obviamente, la mencionada democracia. En un pensamiento agonístico, estos conceptos se oponen al campo semántico del "Otro" que gira alrededor de términos como la barbarie, el despotismo, la esclavitud, la piel no-blanca u oriente. En conjunto, se suman para establecer una base de la identidad europea que refiere a cuatro mitos fundacionales de Europa:

\begin{abstract}
"First, the supremacy of a legal system based on Roman law; second, an ethos of social solidarity and common understanding based on Christian piety and humanism; third, a democratic order rooted in recognition of the rights and freedoms of the individual; and fourth, a universalism based on Reason and other Enlightenment principles of cosmopolitan belonging ${ }^{10} . "$ (Amin 2004, 2)
\end{abstract}

A diferencia con los estados-nación, Europa no goza de un reconocimiento compartido y claramente definido; el discurso hegemónico acerca de Europa y todo lo que implican las identidades europeas es profundamente controvertido (Quenzel 2005). Como Europa es un constructo discursivo sin funciones de estado-nación completas ni fronteras definidas, diferentes ideas y dimensiones son objeto de discusión permanente en los medios de comunicación y el sistema político (Wiener 1998). Esto se refleja también en el debate científico, donde, por ejemplo, Nissen (2006) entiende las identidades europeas como identidades colectivas de los ciudadanos de la Unión frente a las instituciones europeas. Por otro lado, Loth (2002) las interpreta como una opción flexible de identificarse a través de tradiciones compartidas. En su contextualización de Europa, Bach (2000) se refiere primordialmente a las élites burocráticas y administrativas que construyen redes transnacionales que reestructuran las administraciones de los estados miembros. Estas discusiones se pueden resumir en el aspecto que destacan Punscher Riekmann y Wodak (2003), al señalar que las identidades europeas son solamente un estrato o una capa de las muchas posibles para unir una determinada comunidad.

\section{DisCUSIÓN EMPÍRICA - LA PARTICIPACIÓN DE EXTRANJEROS}

Si volvemos al caso de las expresiones políticas de los extranjeros en la costa alicantina, se puede observar que los ciudadanos europeos participan en una gran variedad de aspectos que incluyen entre otros la participación en cuestiones sociales, aspectos políticos informales y también la representación formal a través de concejales electos (Janoschka 2008). A posteriori, la participación se focaliza primordialmente en el uso del voto activo y las transformaciones políticas que se han generado a través de la implicación en la política formal y representativa. Cabe resaltar que en un número creciente de municipios se ha producido tanto el despliegue de partidos locales fundados y dirigidos por extranjeros como la participación en los partidos ya establecidos. En este sentido, se pueden diferenciar cuatro escenarios concurrentes que definen hasta el momento la participación de los extranjeros en la política local:

1. Extranjeros como "atrapa-votos". En algunos municipios, la inclusión de los residentes europeos en las listas de los partidos establecidos (sobre todo, en las listas "conservadoras") ha seguido la pauta de concentrar y atrapar los votos de los extranjeros para estabilizar el poder de la élite política local. Este aspecto corresponde con aspectos de participación "étnica" observada en algunos municipios franceses como Lille (Garbaye 2004). La inclusión política de extranjeros bien conocidos entre la población europea, puede interpretarse como la adaptación de los regímenes locales a las nuevas reglas del juego político en una sociedad "europea". A los concejales extranjeros, se les otorga la función de mejorar la comunicación con los extranjeros, en 
muchos casos a través de nuevas oficinas de atención al residente europeo. Pero estos concejales no forman parte de las redes de actuación en la política local y su papel queda reducido a implementar mejoras directamente vinculadas con la gestión municipal en asuntos que tienen que ver con la relación municipio-residente extranjero.

\section{Extranjeros como "catalizadores de la moderniza-} ción". Muchos pueblos de menor tamaño, primordialmente del interior de la provincia de Alicante, cuentan con una participación política de extranjeros que les da una llave para el poder municipal. En algunos de esos pueblos, donde la cifra de extranjeros es notablemente más alta que la de españoles, la participación de los residentes europeos ha transformado de manera significativa las mayorías políticas. En estos casos, los concejales extranjeros son conscientes de que el gobierno municipal está de cierto modo en sus manos, lo que les lleva a cambiar algunos asuntos clave en la gestión del municipio, entre los que destacan en muchos casos las políticas urbanísticas y las infraestructuras municipales. Más allá de ese aspecto, su participación conlleva también cierta modernización, sobre todo cuando se trata de municipios de menor tamaño. El siguiente ejemplo demuestra la percepción que de la situación antes expuesta pueden tener los extranjeros:

"Esto no es una coalición. Yo suelo estar con el PP, pero puedo fallar y unirme con los otros o estar en contra de un plan. $Y$ entonces, le digo al alcalde: '¿Cómo has llegado al poder?', porque antes hubo aquí cuatro del PSOE, dos del PP y uno del Bloc. Desde que he participado con un amigo, y hemos visitado a todos los extranjeros personalmente, cosa que nunca nadie había hecho, esto se ha cambiado. ¿De dónde provienen todos estos votos, que han ido al PP? Esto son los extranjeros que me han votado a mi, porque he ganado su confianza. Si entonces se hacen cosas que ellos no quieren, ¿qué crees que harán la próxima vez? Harán su propio partido, y 800 o 900 extranjeros que pueden votar y 500 españoles, pues, sería fácil tener la mayoría y tomar el gobierno, y entonces aquí los españoles no mandarán a nadie." (Entrevista con un concejal extranjero).

\section{Extranjeros como "catalizadores de conflictos cul-} turales". En localidades de mayor tamaño, la situación se presenta de una manera muy diferente. Es cierto que en municipios como Dénia o Jávea, que tienen más de 30.000 habitantes, de los cuales más de un tercio son extranjeros de la UE con derecho de voto, el panorama político se ha transformado sustancialmente, sea por la aparición de partidos de extranjeros o por la participación de extranjeros en los partidos tradicionales. Ambos municipios son referentes de importancia para la expresión de identidades valencianas y el partido nacionalista obtiene una representación importante en los parlamentos locales. En este sentido, la cuestión idiomática presenta un aspecto de mayor importancia y conlleva conflictos entre las personas nacidas en la región y los extranjeros. Por ejemplo, durante la legislatura 2003-2007 y en un tiempo en el que tres concejales extranjeros formaban parte del gobierno de Jávea, se produjeron una serie de polémicas acerca del uso de idiomas en actos públicos. Mientras una parte importante de los políticos locales apoyan el uso del valenciano como un derecho constitucional y progresista, muchos extranjeros, cuyo horizonte de interpretación es claramente distinto, consideran la cuestión lingüistica como un obstáculo que les impide la participación en igualdad de condiciones. Habitualmente les cuesta expresarse en castellano, por lo que interpretan el uso del valenciano como una exclusión explícita. En este sentido, se ha producido un bloqueo mutuo de los debates acerca de los posibles contenidos de la Agenda Local 21 durante varios años por la imposibilidad de concertar un idioma para llevar adelante las reuniones. Cuando algunos de los políticos nacionalistas se negaban a usar el castellano, los extranjeros empezaban a debatir en inglés. En resumen, el ejemplo muestra que en los municipios de mayor tamaño, la política local es lo suficientemente profesionalizada como para que los extranjeros tengan que superar barreras inmensas para poder hacerse oír entre los políticos. Los poderes establecidos defienden el acceso a los recursos con firmeza y a través del uso de capital simbólico. A pesar de que el conflicto mencionado surge por el uso del idioma, no es necesariamente el único aspecto de importancia: Favell (2008) ha encontrado en un estudio en Ámsterdam una gama variada de aspectos de índole simbólica que impiden a los extranjeros alcanzar poder político, incluso en casos de un dominio perfecto del idioma local.

4. Extranjeros como "poder político integrado e integral". A pesar de la creciente importancia de la participación política de extranjeros europeos a nivel municipal, el cuarto caso todavía presenta una situación que es poco común, tanto en España como en otros paises de la UE (Garbaye 2004). Uno de los casos excepcionales es el mu- 
nicipio de Teulada-Moraira que no solamente cuenta con uno de los porcentajes de extranjeros más altos de todo el país (casi dos tercios en 2008), sino también con una alta participación municipal de los extranjeros. Ese municipio costero alicantino con actualmente poco más de 14.000 habitantes ha sido un caso paradigmático porque un partido local ha entablado una estrategia común con los residentes europeos para alcanzar el poder político. Desde el año 1999, cuando por primera vez los extranjeros pudieron votar, la lista de ese partido local (que a partir del 2003 se integró bajo las siglas del Partido Popular) se ha aproximado a la paridad. Entre locales, extranjeros y foráneos (en este caso, nuevos residentes provenientes de Madrid) se han repartido las concejalías en función de la calificación de los políticos y, por ejemplo, los extranjeros han ocupado puestos como la concejalía de Medio Ambiente, la concejalía de Hacienda e incluso la vice-alcaldía. Durante la época del gran auge inmobiliario de principios de la década, el consistorio local ha parado los proyectos de inversión urbanistica para desarrollar un Plan General de Ordenación Urbana a través de un proceso participativo de Agenda Local 21. En un momento en el que muchos municipios en Alicante permitían Planes de Actuación Urbanistica de manera desenfrenada, se ha optado por reducir la construcción y establecer medidas de contención a algunas de las "locuras" del boom. Éstas fueron las causas desencadenantes de una movilización de extranjeros comunitarios sin precedentes, en la Comunidad Valenciana y en otras partes de España, para protestar frente al Parlamento Europeo por los Abusos Urbanísticos (Janoschka 2008). Parece interesante evaluar de forma más detallada las opiniones de los políticos involucrados en ese cambio drástico que vivió el municipio de Teulada-Moraira:

\footnotetext{
"Vamos a ver, yo no estaría aquí donde estoy si no creyese, o no estaría donde estoy, con un grupo de ciudadanos europeos participando en la política local. Y de hecho, aquí sabes que tiene responsabilidades como puede ser hacienda, como puede ser el medio ambiente o como puede ser bienestar social, por ponerte algunos ejemplos, que están en manos de concejales que no son españoles. Entonces, en eso hemos creído siempre y yo he creído desde el primer momento. Quizá porque nací aquí. Cuando nací, bueno, todavía no, pero en mi juventud, en mi adolescencia estaba ya todo el boom turístico, estás acostumbrado a relacionarte con gente que viene de otros paises." (J.C., ex-alcalde de Teulada-Moraira)
}

El alcalde electo en el año 1999 (cargo que ocupó hasta marzo de 2009) es consciente de que su capital político central eran las opiniones y la fuerza de voto de los residentes extranjeros. Cambió de manera drástica la administración local e introdujo una asistencia en los idiomas más importantes, que incluyen no solamente el castellano, valenciano e inglés, sino también alemán, francés $y$, en algunas cuestiones, también el holandés. La participación no solamente se refería a la activación del voto extranjero: a posteriori se establecieron diferentes comisiones temáticas que buscaban una gestión "cercana" con la población internacional que habita el municipio. A diferencia de otras localidades, hubo un receso significativo de las tensiones y desapareció una gran parte de las interpretaciones culturales que se emplean a menudo en otros municipios como se ha visto en los apartados anteriores. Como se ha explicado, los políticos han sido conscientes de que la participación conlleva una situación de mutua ganancia y por ello se han asumido estrategias que discursivamente recurren a ideas de una Europa práctica y adaptada en el ámbito local. La siguiente cita refleja esa situación ejemplar:

"Es que aquí tenemos una pequeña Europa y la idea de Europa es lo que nosotros tenemos aquí. Integración total, ¿no? $Y$ todos trabajamos conjuntamente para mejorar nuestra parcela donde vivimos, nuestra zona. Eso es lo que tenía que hacer Europa. Pero, ten en cuenta que nosotros lo que queríamos era hacer eso: nosotros somos una pequeña Europa." (S.T., ex-vice-alcaldesa de Teulada-Moraira)

El recurso a las visiones de una Europa "vivida" demuestra cómo el significado de Europa es capaz de ser utilizado como paraguas discursivo en una sociedad integradora. A diferencia de otros municipios, en Teulada el principio de realizar y construir "una Europa pequeña" es una construcción compartida entre los políticos y los residentes extranjeros, valorada de forma positiva. En consecuencia, se ve que los conflictos entre distintos grupos (sean extranjeros o no) se han canalizado hasta transformarse en una gran componente de construcción social positiva. El uso de, y el recurso común a, las identidades europeas, expresadas por los usos de diferentes idiomas y la implicación activa y consecuente de la población extranjera, establece nuevas relaciones de stakeholder. No es nada fácil, pues más allá de requerir las mentes abiertas también exige un compromiso de transformar la forma de hacer la política en su dimensión cotidiana y de compartir los recursos políti- 
cos con grupos que históricamente no han sido evaluados como una parte activa de la ciudadanía.

\section{INTERPRETACIÓN FINAL Y CONCLUSIONES}

Esta contribución persiguió dos metas centrales. Por un lado, trataba de abordar la elaboración y el debate de un cuadro teórico apto para analizar procesos de participación política relativamente nuevos en la Unión Europea y en España, procesos que son una expresión práctica de una concepción de "Europa" que fue ideada a lo largo de las últimas décadas por los políticos y otros representantes de los respectivos estados miembros. Por otro lado, en el artículo se quiso debatir cómo esta práctica de una Europa sin fronteras se lleva a cabo en el ámbito de las políticas locales y cuáles son los nuevos retos frente a procesos de integración que diluyen las diferencias claras y marcadas entre, expresado de forma coloquial, "los de aquí de siempre" y nuevos residentes, que en el caso estudiado son ciudadanos de la UE, mayores de edad y que en su mayoría no han llegado para trabajar, sino con el fin de vivir de las rentas de su vida laboral ya finalizada. Muchos individuos de este grupo, que en su composición es muy específico, pero cada vez más numeroso, tienen el tiempo y la vocación de participar en cuestiones políticas - un aspecto que hace extremadamente interesante el estudio de su participación-. A través del debate defendido en esta aportación, se pueden derivar tres conclusiones centrales.

Primera, desde un punto de vista teórico-conceptual, la participación política de extranjeros en las zonas del litoral español se puede interpretar como una práctica de ciudadanía de la Unión Europa de libre movimiento, donde los individuos se apropian de forma activa de las posibilidades, las libertades y las garantías que les concede el derecho comunitario. Es una situación ejemplar y destacadamente positiva que las personas que gozan de los derechos brindados por el marco legal de la Unión Europea, no solamente hagan un uso activo de ello sino que también generen recursos de identidad que se refieren al sueño de una sociedad europea integradora. En este sentido, la propuesta conceptual de pensar la ciudadanía como una práctica de identidades se ve reflejada incluso en la vida diaria de los ciudadanos de la Unión Europea que se han mudado al litoral español.
Segunda, a pesar de que algunos aspectos de la participación política son teóricamente positivos y comportan fragmentos de una sociedad integradora, no conviene adoptar ninguna perspectiva demasiada inocente. La política es primordialmente una lucha por cuestiones de poder que conlleva un sinfín de conflictos. En este sentido, la participación de extranjeros se puede interpretar como una expresión de disputas y querellas en la cual los poderes tradicionalmente establecidos no quieren perder su influencia y su posición dominante. Frente a las amenazas que significa la introducción de nuevos participantes en el juego político local, se pueden dar soluciones e interpretaciones integradoras o excluyentes. Desde un punto normativo, sería un ideal que los extranjeros pudieran participar de manera igual y equitativa. Pero en la "realidad", es más común que se den interpretaciones restrictivas basadas en aspectos culturales y en la expresión de derechos arcaicos y anticuados. En este sentido, es importante proseguir en la investigación de los conflictos que puedan surgir de la participación de los extranjeros e inmigrantes, ya que éstos se pueden interpretar como un laboratorio social paradigmático.

Finalmente, el ejemplo del primer alcalde inglés en España que se expuso al principio también es susceptible de dos lecturas posibles. Por un lado, es un signo de una transformación que vivió España a lo largo de la primera década del siglo XXI y que se profundizará en el futuro cercano. Las posiciones de poder político local están cambiando profundamente a causa de la integración de los inmigrantes. En este sentido, será cada vez más habitual ver reflejado en la composición de los consistorios locales el camino a la sociedad migratoria que España emprendió desde mediados de los años 1990. A partir del año 2011, cuando la inmensa mayoría de los inmigrantes puedan votar en los comicios municipales, será habitual ver concejales e incluso algunos alcaldes de procedencia extranjera en los ayuntamientos del país. Por otro lado, el ejemplo del primer alcalde británico también tiene un segundo sentido. Se produjo en un momento en el que en España y en el mundo entero estalló la burbuja financiera y su surgimiento en el tiempo obedeció al conocimiento público de unos escándalos de corrupción urbanística. Muchos de los residentes europeos que participan en la política en el Levante español han levantado su voz motivados explícitamente por los excesos de un urbanismo que, según informes de organismos internacionales como Greenpeace o Transparency International, 
ha sido calificado como una locura o avalancha urbanistica que ha destrozado una buena parte del litoral del pais. Esperemos que la necesaria reflexión en el seno de la crisis actual no solamente cambie algunos de los excesos de la década pasada. Sería idóneo plantear que la política local vuelva a ser la expresión de los deseos y de los planteamientos de la sociedad que habita ese espacio delimitado, en lugar de ser un espacio de apropiación de los recursos de todos, por lo menos de forma ilícita desde un punto de vista filosófico. Si se toma el mencionado aspecto en serio, la política local sería diferente después de la crisis, ya que como resultado de los procesos migratorios masivos de la última década tendrá que responder e integrar también los deseos y las necesidades de los recién llegados.

\section{NOTAS}

* Esta investigación está financiada por un Postdoctoral Marie Curie Intra-European-Fellowship, dentro del VII Programa Marco de la Comisión Europea (PIEF-GA-2008-220287). Se realiza en el Centro de Ciencias Humanas y Sociales del CSIC, cuyo entorno ha sido un factor estimulante en la elaboración y redacción de ese artículo. Agradezco los comentarios y las críticas recibidas a una versión preliminar del texto, que se presentó a debate en el marco de las /I Jornadas sobre Politicas migratorias, justicia y ciudadanía, celebradas en noviembre de 2008 en el CCHS-CSIC. Remito un agradecimiento especial a Victoria Fernández, Silvia Marcu, Vicente Rodríguez y Juan Carlos Velasco, cuyas lecturas críticas del manuscrito han contribuido a perfilar el análisis.

1 La Vanguardia, 31 de octubre de 2008, edición digital: http://www. lavanguardia.es//v24h/20081031/53 570519254.html, 24 de noviembre de 2008)

2 Véase, entre otros, el diario El Mundo (http://www.elmundo.es/elmundo/2008/10/31/valencia/1225480690. html, 24 de noviembre de 2008) o ABC (http://www.abc.es/20081102/ nacional-nacional/sainete-fulgencio20081102.html, 24 de noviembre de 2008).

Recibido: 15 de junio de 2009

Aceptado: 10 de julio de 2009
3 Véase, entre otros, el Daily Telegraph (http://www.telegraph.co.uk/news/ worldnews/europe/spain/3286974/ British-expat-becomes-accidental-mayor-of-Costa-Blanca.html, 24 de noviembre de 2008), el Mirror (http://www.mirror.co.uk/news/topstories/2008/10/31/brit-becomesaccidental-mayor-of-costa-blanca115875-20856459/, 24 de noviembre de 2008) o la $B B C$ (http://news.bbc. co.uk/1/hi/world/europe/7701551. stm, 25 de noviembre de 2008).

4 Tanto los parágrafos del 17 al 21 del Tratado de Maastricht como la Directiva 94/80/EG establecen el derecho de voto activo y pasivo en elecciones municipales a todos los ciudadanos de la Unión Europea que viven en un país miembro de la UE. España adaptó ese reglamento con un cambio legislativo en el mes de mayo de 1997, que hizo posible la participación de los extranjeros comunitarios a partir de los comicios municipales del año 1999.

5 El Padrón Municipal del 1 de enero de 2008 suma 5,27 millones de extranjeros que viven en el país, un 11,4\% de la población total de España (INE 2008).

6 Desde el año 2004, se han efectuado más de 90 entrevistas en profundidad a una gran variedad de actores políticos en municipios de la Comunidad Valenciana y en Andalucía.

7 Original en inglés. Traducción: "Para poder existir, identidad requiere diferencia $y$, para asegurar su propia certeza, la convierte en otredad." 
8 Entrevista original en inglés. Traducción de la cita: "Como alguien que viene de Gran Bretaña y alguien que trabajaba en un gobierno local en Gran Bretaña, llevo un tipo de concepto europeo de democracia y decencia, y consulta y justicia y confianza y todo eso. También en Gran Bretaña, uno no puede siempre confiarse en los políticos. Pero, si tratas aquí con ellos durante un tiempo, lo que haces es que tienes que limpiar tu cabeza de todo tipo de idea que llevas dentro, de Inglaterra, Alemania u Holanda, de donde eres, y empezar a pensar en español, porque ellos no aceptan o no entienden ninguno de esos principios."

9 Texto original en inglés. Traducción de la cita: "un lugar de pertenencia plural y extraña que se dibuja en las variadas geografías de formación cultural".

10 Texto original en inglés. Traducción de la cita: "Primero, la supremacía de un sistema legal basado en el derecho romano; segundo, una ética de solidaridad social y sentido común basado en el humanismo y la piedad cristiana; tercero, un orden democrático arraigado en los derechos y las libertades del individuo; y cuarto, un universalismo basado en la razón y otros principios de pertenencia cosmopolita derivados de la época ilustrada."

\section{BIBLIOGRAFÍA}

Amin, A. (2004): Multi-ethnicity and the Idea of Europe, en: Theory, Culture and Society, 21, 2, 1-24.

Anderson, B. (2006) [1983]: Imagined Communities: Reflections on the Origin and Spread of Nationalism, Londres.

Arendt, H. (1987): La decadencia de la nación-Estado y el final de los derechos del hombre, en: Los orígenes del totalitarismo, vol. 2, Madrid, 392-438.

Bach, M. (2000): Die Europäisierung der nationalen Gesellschaft? Problemstellungen und Perspektiven einer Soziologie der europäischen Integration, en: Bach, M. (ed.): Die Europäisierung nationaler Gesellschaften, Wiesbanden, 11-35.

Barnett, C. (2003): Culture and Democracy. Media, Space and Representation, Edimburgo.

Bauböck, R.; Mokre, M. y Weiss, G. (2003): Nun wächst zusammen, was noch nie zusammen gehörte, en: Mokre, M.; Weiss, G.; Bauböck, R. (eds.): Europas Identitäten. Mythen, Konflikte, Konstruktionen, Fráncfort, 9-21.

Bauböck, R. (2005): Expansive Citizenship Voting beyond Territory and Membership, en: Political Science \&t Politics, 38: 683-687.

Beaverstock, J. (2005): Transnational Elites in the City: British Highly-Skilled Inter-Company Transferees in New York City's Financial District, en: Journal of Ethnic and Migration Studies, 31, 245-268.

Benhabib, S. (2005): Los derechos de los otros. Extranjeros, residentes y ciudadanos, Barcelona.

Benson, M. y O'Reilly, K. (2009): Migration and the search for a better way of life: a critical exploration of lifestyle migration, en: The Sociological Review, 57, en prensa.

Bhabha, H. (2008): Locations of Culture, en: Khagram, S.; Levitt, P. (eds.): The Transnational Studies Reader. Intersections and Innovations, Londres/ Nueva York, 333-338.

Butler, J. (1990): Gender Trouble, Londres/ Nueva York.

Castiglione, D. (2009): Political identity in a community of strangers, en: Checkel, J.; Katzenstein, P. (eds.): European Identity, Cambridge, 29-51.
Checkel, J. y Katzenstein, P. (2009): The politicization of European identities, en: Checkel, J.; Katzenstein, P. (eds.): European Identity, Cambridge, 1-25.

Connolly, W. (2002): Identity|Difference: Democratic Negotiations of Political Paradox, Expanded Edition, Minneapolis.

Davidson, A. (2000): Fractured Identities: Citizenship in a Global World. en: Vasta, E. (ed.): Citizenship, Community and Democracy, Londres, pp. 3-21.

Day, S. y Shaw, J. (2002): European Union Electoral Rights and the Political Participation of Migrants in Host Polities, en: International Journal of Population Geography, 8: 183-199.

Della Porta, D. y Tarrow, S. (eds., 2004): Transnational Protest and Global Activism. People, Passions, and Power, Rowman \& Littlefield, New York.

Durán, R. y Martín, M. (2008): La integración política de los inmigrantes. La vía de sufragio, Granada.

Echezarreta, M. (2005, ed.): El Lugar Europeo de Retiro. Indicadores de excelencia para administrar la gerontoinmigración de ciudadanos de la Unión Europea en municipios españoles, Granada.

Eder, K. (2006): Europe's Borders. The Narrative Construction of the Boundaries of Europe. en: European Journal of Social Theory, 9, 255-271.

Faist, T. (1998): International Migration and Transnational Social Spaces, en: Archives Européennes de sociologie / European Journal of Sociology, 39: 213-247.

Favell, A. (2003): Games without Frontiers? Questioning the Transnational Social Power of Migrants in Europe, en: Archives Européennes de Sociologie / European Journal of Sociology, 44: 397-427.

- (2008): Eurostars and Eurocities: Free Movement and Mobility in an Integrating Europe, Malden. 
Featherstone, M. (1991): Consumer Culture and Postmodernism, Londres.

Finke, B. y Knodt, M. (2005): Einleitung: Zivilgesellschaft und zivilgesellschaftliche Akteure in der Europäischen Union. en: Knodt, M. y Finke, B. (eds.): Europäische Zivilgesellschaft. Konzepte, Akteure, Strategien, Wiesbaden, 11-28.

Garbaye, R. (2004): Ethnic Minority Local Councillors in French and British Cities: Social Determinants and Political Opportunity Structures. en: Penninx, R. et al. (eds.): Citizenship in European cities. Immigrants, local politics and integration politics, Aldershot, 39-56.

García, M. (2006): Citizenship Practices and Urban Governance in European Cities, en: Urban Studies, 43: 745-765.

Glasze, G. (2007): The Discursive Construction of a World-Spanning Region and the Role of Empty Signifiers: The Case of Francophonia, en: Geopolitics, 12: 656-679.

Grundy, S. y Jamieson, L. (2007): European Identities: From Absent-Minded Citizens to Passionate, en: European Sociology, 41, 663-680.

Habermas, J. (1999): La inclusión del otro, Barcelona.

- (2009): ¡Ay, Europa! Pequeños escritos políticos, Madrid.

Hall, S. (1996): Introduction: Who Needs "Identity"?, en: Hall, S.; Du Gay, P. (eds.): Questions of Cultural Identity, 1-17. London.

- (1997): The Spectacle of the Other. en: Hall, S. (ed.) Representation. Cultural Representations and Signifying Practices, Londres, 223-290.

Harvey, D. (2006): The geographies of critical geography, en: Transactions of the Institute of British Geographers, 31: 409-412.

Hermes, J. y Dahlgren, P. (2006): Cultural studies and citizenship, en: European
Journal of Cultural Studies, 9: 259596.

Herrmann, R. y Brewer, M. (2004): Identities and Institutions. Becoming European in the EU, en: Herrmann, R.; Risse, T.; Brewer, M. (eds.): Transnational identities. Becoming European in the EU, Lanham, 1-23.

INE (Instituto Nacional de Estadisticas, 2008) Padrón Municipal al 1 de enero de 2008, Madrid, Información en línea: www.ine.es, 24 de noviembre de 2008.

Isin, E. y Wood, P. (1999): Citizenship and Identity, Londres.

Jackson, P.; Crang, P. y Dwyer, C. (2004): Introduction. The Spaces of Transnationality, en: Jackson, P.; Crang, P.; Dwyer, C. (eds.): Transnational Spaces, Londres/Nueva York, 1-23.

Janoschka, M. (2008): Identity Politics as an Expression of European Citizenship Practice: Participation of Transnational Migrants in Local Political Conflicts, en: Anghel, R. et al. (eds.): The Making of World Society. Perspectives from Transnational Research, Bielefeld, 133-152.

Jennings, M. K. y Markus, G. B. (1988): Political Involvement in the Later Years: A Longitudinal Survey, en: American Journal of Political Science, 32: 302316.

Kantner, C. (2006): Collective Identity as Shared Ethical Self-Understanding. The Case of the Emerging European Identity, en: European Journal of Social Theory, 9, 501-523.

King, R.; Warnes, A. y Williams, A. (2000): Sunset Lives. British Retirement Migration to the Mediterranean, Oxford.

Laffan, B. (2004): The European Union and Its Institutions as "Identity Builders", en: Herrmann, R.; Risse, T.; Brewer, M. (eds.): Transnational identities. Becoming European in the EU, Lanham, 75-96.
Lepofsky, J. y Fraser, J. (2003): Building Community Citizens: Claiming the Right to Place-Making in the City, en: Urban Studies, 40: 127-142.

Loth, W. (2002): Die Mehrschichtigkeit der Identitätsbildung in Europa. Nationale, regionale und europäische Identität im Wandel. en: Elm, R. (ed.): Europäische Identität: Paradigmen und Methodenfragen, Baden-Baden, 93-110.

Low, M. (2004): Cities as Spaces of Democracy: Complexity, Scale and Governance, en: Barnett, C.; Low, M. (eds.): Spaces of Democracy. Geographical Perspectives on Citizenship, Participation and Representation, Londres, 129-146.

Marshall, T. H. (1950): Citizenship and social class and other essays, Cambridge.

Marti, M. y Bonet, J. (2008): Los movimientos urbanos: De la identidad a la globalidad, en: Scripta Nova (http://www. ub.es/geocrit/-xcol/403.html).

Massey, D. (2004): Geographies of Responsibility, en Geografiska Annaler, 86: 5-18.

Mclntyre, N. (2009): Re-thinking Amenity Migration: Integrating Mobility, Lifestyle and Social-Ecological Systems. en: Die Erde, 40, 229-250.

Mikkeli, H. (1998): Europe as an idea and an identity, Houndmills.

Mitchell, K. (2003): Educating the National Citizen in Neoliberal Times: from the Multicultural Self to the Strategic Cosmopolitan, en: Transactions of the Institute of British Geographers, 28: 387-403.

- (2007): Geographies of identity: the intimate cosmopolitan, en: Progress in Human Geography, 31: 706-720.

Mouffe, C. (2002): Which Kind of Public Space for a Democratic Habitus?, en: Hillier, J.; Rooksby, E. (eds.): Habitus: A Sense of Place. Second Edition, Aldershot, 109-116. 
MTAS (Ministerio de Trabajo y Asuntos Sociales, 2007): Plan estratégico Ciudadanía e Integración 2007-2010, Documento en línea: http://www.mtas. es/es/migraciones/Integracion/PlanEstrategico/Docs/PECIDEF180407.pdf, 24 de noviembre de 2008.

Nicholls, W. (2009): Place, networks, space: theorising the geographies of social movements, en: Transactions of the Institute of British Geographers, 34: 78-93.

Nijman, J. (2007): Locals, exiles and cosmopolitans: a theoretical argument about identity and place in Miami, en: Tijdschrift voor economische en sociale geografie, 98: 176-187.

Nissen, S. (2006): European Identity and the Future of Europe, en: Bach, M.; Lahusen, C; Vobruba, G. (eds.): Europe in Motion. Social Dynamics and Political Institutions in an Enlarging Europe, Berlín, 155-174.

Oliver, C. (2007): Retirement Migration. Paradoxes of Ageing, Nueva York.

Ong, A. (2006): Mutations in Citizenship, en Theory, Culture \&t Society, 23: 499531.

Painter, J. (2008): European Citizenship and the Regions, en: European Urban and Regional Studies, 15: 5-19.

Penninx, R.; Kraal, K.; Martiniello, M. y Vertovec, S. (2004): Citizenship in European Cities: Immigrants, Local Politics and Integration Policies, Aldershot.

Penrose, J. y Mole, R. (2008): Nation-States and National Identity, en: Cox, K.; Low,
M.; Robinson, J. (eds.): The SAGE Handbook of Political Geography, Londres, 271-283.

Punscher Riekmann, S. y Wodak, R. (2003): Europe for all - diskursive Konstruktionen europäischer Identitäten, en: Mokre, M.; Weiss, G.; Bauböck, R. (eds.) Europas Identitäten. Mythen, Konflikte, Konstruktionen, Fráncfort/ Nueva York, 283-303.

Quenzel, G. (2005): Konstruktionen von Europa. Die europäische Identität und die Kulturpolitik der Europäischen Union, Bielefeld.

Rodríguez, V.; Casado, M. A. y Huber, A. (2005): La migración de jubilados europeos en España, Madrid.

Rose, J. (2001): Contexts of Interpretation: Assessing Immigrant Reception in Richmond, Canada, en: The Canadian Geographer, 45: 474-493.

Routledge, P. (2008): Transnational Political Movements, en: Cox, K.; Low, M.; Robinson, J. (eds.): The SAGE Handbook of Political Geography, Londres, 335-349.

Rumford, C. (2002): European Civil Society or Transnational Social Space? Conceptions of Society in Discourses of EU Citizenship, Governance and the Democratic Deficit: an Emerging Agenda, en: European Journal of Social Theory, 6: 25-43.

Said, E. (2003): Orientalismo, Barcelona.

Sassen, S. (1999): La ciudad global. Nueva York, Londres, Tokyo, Buenos Aires.
Schriewer, K. y Encinas, I. (2008): Being Misleading About Where One Resides. European Affluence Mobility and Registration Patterns, en: Ethnologia Europaea - Journal of European Ethnology, 37, 98-106.

Shachar, A. y Hirschl, R. (2007): Citizenship as Inherited Property, en: Political Theory, 35: 253-287.

Smith, M. P. (2001): Transnational Urbanism: locating globalization, 0xford.

Urry, J. (2000): Sociology Beyond Societies: Mobilities for the Twenty-First Century, Londres.

Vandenberg, A. (2000): Contesting Citizenship and Democracy in a Global Era, en: Vandenberg, A. (ed.): Citizenship and Democracy in a Global Era, Londres/Nueva York, 3-17.

Warnes, A. (2004, ed.): Older Migrants in Europe. Essays, Projects and Sources, Sheffield.

Wiener, A. (1998): "European" Citizenship Practice. Building Institutions of a Non-State, Oxford.

Wodak, R. (2004): National and Transnational Identities: European and Other Identities Con-structed in Interviews with EU Officials. en: Herrmann, R.; Risse, T.; Brewer, M. (eds.): Transnational identities. Becoming European in the EU, Lanham, 97-127.

Wodak, R. et al. (1999): The Discoursive Construction of National Identities, Edimburgo. 